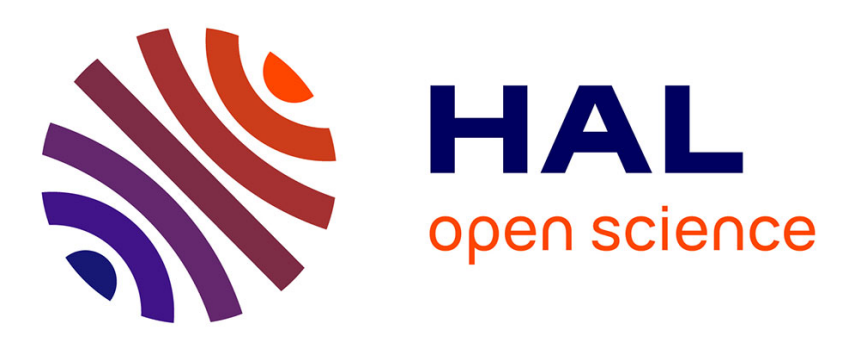

\title{
A Multivariate Treatment of Bias for Sequential Data Assimilation: Application to the Tropical Oceans
}

\author{
Magdalena Balmaseda, Dick Dee, Arthur Vidard, David P. Anderson
}

\section{To cite this version:}

Magdalena Balmaseda, Dick Dee, Arthur Vidard, David P. Anderson. A Multivariate Treatment of Bias for Sequential Data Assimilation: Application to the Tropical Oceans. Quarterly Journal of the Royal Meteorological Society, 2007, 133 (622), pp.167-179. 10.1002/qj.12 . inria-00176159

\section{HAL Id: inria-00176159 \\ https://hal.inria.fr/inria-00176159}

Submitted on 2 Oct 2007

HAL is a multi-disciplinary open access archive for the deposit and dissemination of scientific research documents, whether they are published or not. The documents may come from teaching and research institutions in France or abroad, or from public or private research centers.
L'archive ouverte pluridisciplinaire HAL, est destinée au dépôt et à la diffusion de documents scientifiques de niveau recherche, publiés ou non, émanant des établissements d'enseignement et de recherche français ou étrangers, des laboratoires publics ou privés. 


\title{
A Multivariate Treatment of Bias for Sequential Data Assimilation: Application to the Tropical Oceans
}

\author{
By M.A. Balmaseda* ,D. Dee, A. Vidard and D.L.T. Anderson \\ $E C M W F, U K$
}

(Received 30th August 2005; revised July 2006; final version Oct 2006 )

\section{Summary}

This paper discusses the problems arising from the presence of system bias in ocean data assimilation taking examples from the ECMWF ocean reanalysis used for seasonal forecasting. The examples illustrate how in a biased system, the non-stationary nature of the observing system is a handicap for the reliable representation of climate variability. It is also shown how the bias can be aggravated by the assimilation process, as is the case for the temperature bias in the eastern equatorial Pacific, linked to a spurious vertical circulation generated by the data assimilation.

A generalized algorithm for treatment of bias in sequential data assimilation has been implemented. The scheme allows the control variables of the bias to be different from those for the state vector. Experiments were conducted to evaluate the sensitivity of the results to the choice of bias variables. Results highlight the importance of the correct choice of variables for the bias: while correcting the bias in the pressure field reduces the bias in temperature and in the velocity field, the direct correction of the bias in the temperature field reduces the temperature bias, but significantly increases the error in the velocity field.

Analysis of the error statistics reveals that the bias term is not constant in time, but exhibits large interannual fluctuations. The bias algorithm has been generalized further to include temporal variations of the bias term. A memory factor is included to allow for the slow variations of the bias, and a prescribed bias term is added to represent errors known a-priori. Several experiments have been conducted to illustrate the sensitivity of the results to the time evolution of the bias.

KEYWORDS: oceanography ocean reanalysis climate variability

\section{INTRODUCTION}

Ocean data assimilation is a common practice for the generation of historical climate reanalyses used in the study of climate variability (Ji et al. 1995, Carton et al. 2000b, Stammer et al. 2002, among others). It is also used for the initialization of seasonal forecasts with coupled models (Behringer et al. 1998, Alves et al. 2004, Chen et al. 2004, Huddleston et al. 2004). The benefits of data assimilation in reducing the uncertainty in and improving initial conditions for seasonal forecasts have been demonstrated (Alves et al. 2004, Balmaseda 2004, Vidard et al. 2005). However, the procedure itself is not without problems. It is often the case that the magnitude of the time-averaged background error is comparable to the random component, indicating sizeable system bias. System bias is a serious obstacle to the reliable representation of climate variability since, in its presence, a time dependent observing system can induce spurious time variability in the analysis (Segschneider et al. 2000, Balmaseda 2004, Vidard et al. 2005). A deficient data assimilation system may introduce error in the analysis (Burgers et al. 2002, Bell et al. 2004). The problem of bias is not exclusive to the ocean data assimilation systems; it is also an important issue in atmospheric reanalysis (Dee 2005, Dee (2005) in what follows).

Dee and Da Silva (1998) (DdS in what follows) developed an algorithm for the online estimation and correction of the bias in sequential data assimilation. It was successfully applied by Dee and Todling (2000) to the global assimilation of humidity observations in the Goddard Earth Observing System (GEOS) data

* Corresponding author:ECMWF, Reading RG2 9AX, UK,

e-mail: Balmaseda@ecmwf.int

(C) Royal Meteorological Society, 2005. 
assimilation system. The general algorithm was too costly for multivariate bias estimation in a global system, since it required an extra assimilation step to estimate the bias. A simplified version of the algorithm using a single step was first applied by Radakovitch et al. (2001) to land-surface temperature assimilation. For a comprehensive review of the different bias correction algorithms see Dee (2005).

Bell et al. (2004) (BMN in what follows) used the simplified one-step DdS algorithm for the on-line estimation of subsurface temperature bias in the tropical oceans. However, in the BMN scheme the bias correction is not applied directly to the temperature field, but applied as a correction to the pressure gradient. The BMN method was also applied by Huddleston et al. (2004) to diagnose errors in the wind stress forcing. The ideas of the pressure correction have also been developed in parallel outside the field of data assimilation by Sheng et al. (2001), and have been successfully applied to the correction of the Gulf stream representation in eddy-permitting models (Eden et al. 2004).

The simplified one-step DdS algorithm requires proportionality between the bias and state error covariance matrices. In this regard, the BMN scheme deviates from the DdS algorithm. Dee (2005) presents a more general framework, where the bias and the state vector can use different control variables. In this paper we derive the corresponding one-step version of the Dee (2005) generalized algorithm (G1S in what follows), and discuss the approximations required. Although the derivation for th G1S assumes linearity, the final altorithm is also valid for the non linear case. The G1S scheme encompasses naturally the BMN scheme as a specific choice of bias variable. The scheme can also be interpreted as a multivariate bias correction algorithm. The G1S scheme has been implemented in the ECMWF ocean analysis system. Sensitivity experiments have been conducted to assess the impact of the specific choice of bias-variables on the results.

The DdS bias correction algorithm requires the prescription of a model for the time evolution of the bias. The simplest and most widely-used model is that of constant-in-time bias. Dee and Todling (2002) discuss this assumption, pointing out the pitfall that a constant bias allows a single observation to influence the bias estimation indefinitely. The introduction of a memory term may thus be desirable. Moreover, the systematic error may not be constant in time: it may be flow-dependent (e.g. depend on the diurnal or seasonal cycle), or it may be associated with the non-stationary errors of the external forcing (such as discontinuities in the atmospheric analysis system that provides the surface fluxes). In an application to land surface temperature assimilation, Radakovitch et al. (2001) introduced a model for the bias where the diurnal cycle is represented as a harmonic function. Chepurin et al. (2005) formulated a comprehensive model for bias evolution that consists of the online estimation of the multiplicative coefficients associated with given patterns of spatial variability. Although quite general and elegant, the method relies heavily on the robustness and stationarity of the prescribed spatial patterns, and its application to historical reanalysis of the global ocean may be premature (for instance, the patterns of error of subsurface temperature in the southern hemisphere would be difficult to obtain from past records). In this paper we choose a simpler model for the time evolution of the bias term that allows us to discuss the sensitivity of the solution to the prescribed parameters.

Chepurin et al.(2005) deals mainly with the time evolution of the bias, but the multivariate aspects of the bias are not dealt with: the bias is applied directly to the temperature field (observed variable). On the other hand, the BMN scheme, 
sophisticated in its treatment of the bias variables, uses the assumption that the bias is constant in time. The work presented in this paper evaluates the sensitivity of the ocean analysis system to these two aspects of the bias correction algorithm: the choice of bias variables, including the multivariate formulation of the bias covariance matrix, and the model for the time evolution. The emphasis is on the equatorial oceans, in particular the Pacific ocean.

The paper is organized as follows: In section 2, the outstanding problems arising from bias in the system are illustrated with examples from the ECMWF operational ocean analysis systems. Section 3 introduces a generalized one step algorithm for treatment of system bias, based on Dee (2005). The formulation allows the balance relationships in the bias error covariance matrix to be different from those in the state error covariance matrix. It also allows for slow time evolution of the system bias. The sensitivity to the multivariate formulation and to the time evolution is discussed in section 4 . The sensitivity experiments allow us to discuss the merits of the BMN pressure correction scheme versus the standard correction of the bias in the temperature field, as in Chepurin et al.(2005). The experiments also illustrate the importance of limiting the memory of the bias term. A summary and conclusions are given in section 5 .

\section{BIAS IN THE ECMWF OCEAN ANALYSES SYSTEMS}

\section{(a) ECMWF operational ocean data assimilation systems}

ECMWF has had an operational ocean analysis since 1996 as part of the seasonal forecasting system. In January 2001, the data assimilation component of the original operational system (System 1 or S1 in what follows) was upgraded together with other components in the seasonal forecasting suite. We refer to this second operational analysis as System2 (S2), and at the time of writing this is the current operational system. The operational system consists of real-time as well as historical ocean analyses, the latter being used as initial conditions for the hindcasts to calibrate the seasonal forecast system. A description of the two successive ocean analysis systems is given in Balmaseda (2004) and Alves et al. (2004). Here we just offer some concise information.

The background state for the data assimilation is produced by an ocean model forced by analyzed surface fluxes of momentum, heat and fresh water. The ocean model is based on HOPE (Hamburg Ocean Primitive Equations). The ocean data assimilation scheme is an Optimum Interpolation (OI) scheme, and in the results presented here only subsurface temperature data are assimilated. The original system (S1) was univariate, while S2 includes balance constraints to update salinity and velocity, following the schemes proposed by Troccoli et al. (2002) and Burgers et al. (2002) respectively.

Originally, the temperature data came from the GTSPP (Global Temperature Salinity Profiling Project) at NODC (National Oceanographic Data Center). These include data from XBTs, mooring data from TAO, PIRATA and TRITON, and more recently from the ARGO floats. Since 2004, the observations are taken directly from the Global Telecommunication System (GTS). An analysis is performed every 10 days, using observations which span a window five days either side of the model background. There is no temperature assimilation in the top model level; instead the model SST is relaxed to analyzed SST (Reynolds et al. 2002) with a relaxation time-scale of 3 days. 
In this section we will consider four sets of analyses: the operational analyses, called $A_{-} S 1$ and $A \_S 2$ for systems S1 and S2 respectively, and the corresponding control analyses $\left(C_{-} S 1\right.$ and $\left.C_{-} S 2\right)$ without data assimilation. Forcing fields from ERA-15 (Gibson et al., 1997) are used until 1993, and fluxes from the operational atmospheric analysis system after that. ERA-40 (Uppala et al., 2005) was not available at the time of operational implementation of S2, but fluxes from ERA40 will be used in the experiments described in section 4 .

\section{(b) Errors in the mean state}

Figure 1a shows a vertical profile of the 1987-2001 mean difference between the analyses and the observations averaged over the Niño 3 area. The solid line corresponds to $A_{-} S 2$ and the dotted line $C \_S 2$. Below 200 metres, $A \_S 2$ is much less biased than $C_{-} S 2$. In the upper 200 meters both analyses are biased with respect to the observations, although in opposite directions: the analysis without data assimilation is too cold with respect to the observations, while the analysis with data assimilation is too warm. The data assimilation appears to be overcorrecting the model errors. It could also be that the errors in $A_{-} S 2$ analysis are of a different nature to those in $C_{-} S 2$, as if the data assimilation procedure had become a source of error itself.

To assess the impact of the data assimilation it is important to use independent data such as the velocity data provided by the TAO moorings. Figure $1 \mathrm{~b}$ shows the average zonal velocity at mooring location $110^{\circ} \mathrm{W}$. The grey line represents the observations from TAO. The velocities from $A_{-} S 2$ and $C_{-} S 2$ are represented by the solid and dotted lines respectively. The maximum value of the undercurrent is better reproduced by $A_{-} S 2$ than by $C_{-} S 2$, which produces weaker-than-observed currents. However, in the assimilation, the undercurrent is too broad, and does not have a sharp maximum centered around the thermocline as in the observations. The large values of the zonal velocity beneath the thermocline are associated with a spurious downwelling circulation discussed in section 4 . The degradation of the vertical structure of the equatorial currents as a consequence of the assimilation of temperature data is a common feature in other assimilation systems (Burgers et al. 2002, Vialard et al. 2003, Balmaseda 2004, Huddleston et al. 2004, Ricci et al. 2005), although it seems to be absent in 4D-Var analyses (Weaver et al. 2003, Vialard et al. 2003).

BMN suggested that spurious vertical circulations induced by the assimilation may cause additional errors in the temperature field. They went further to suggest a possible positive feedback between errors induced by the data assimilation (degradation of currents) and errors in the model temperature field, that could lead to the existence of a bias in the assimilation system different from the bias in the system without data assimilation. The BMN method is successful in reducing the spurious circulations induced by the data assimilation, and as a consequence, reduces the bias in the temperature field.

Figure 2 shows the time evolution of the temperature increment from $A_{-} S 2$ in the Eastern Pacific (Niño 3 area: $90^{\circ} \mathrm{W}-159^{\circ} \mathrm{W}, 5^{\circ} \mathrm{N}-5^{\circ} \mathrm{S}$ ) at $100 \mathrm{~m}$ depth. The 24-month running mean of the assimilation increment, representative of the low frequency component of the error is shown in black. We equate this component of the error to the bias, since it represents the part of the error that is correlated in time, with time decorrelation scales much larger than the assimilation cycle. The high-pass residuals, representative of the random component of the error, appear in grey. Note that the magnitude of the low frequency component of error is not 

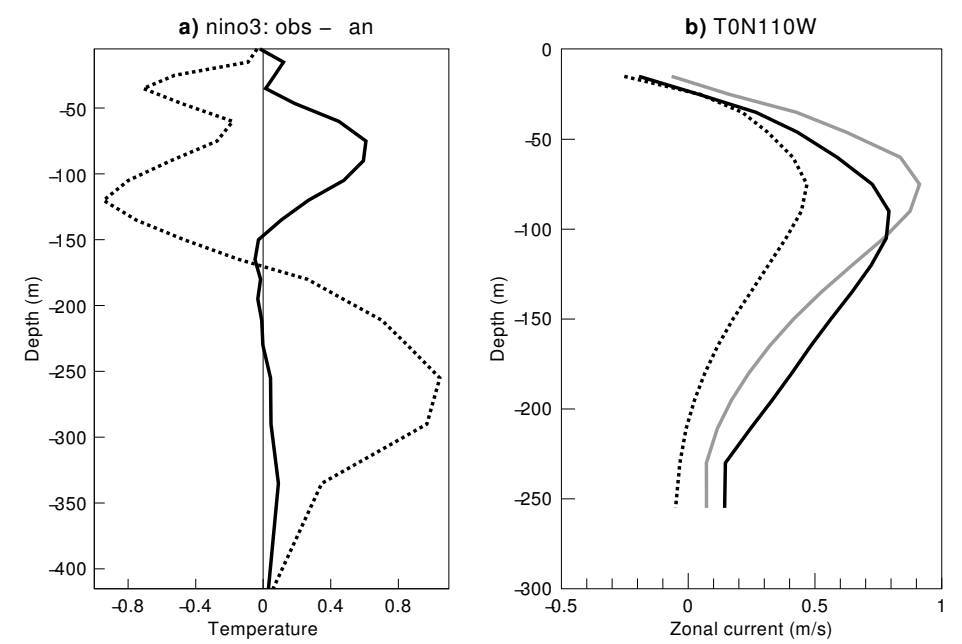

Figure 1. a) Vertical profiles of the 1987-2001 mean temperature statistics "observation minus analysis", averaged over the Eastern Pacific (Niño 3 area). The solid line is for the $A \_S 2$ operational ocean analysis, and the dotted line is for the $C_{-} S 2$. The assimilation of data reverses the sign of the system bias in the upper $150 \mathrm{~m}$. b) Vertical profiles of the $1987-2002$ mean zonal velocity at $110^{\circ} \mathrm{W}$. The grey line represents the TAO current meter measurements. The solid line is for $A_{-} S 2$ and the dotted line is for $C_{-} S 2$.

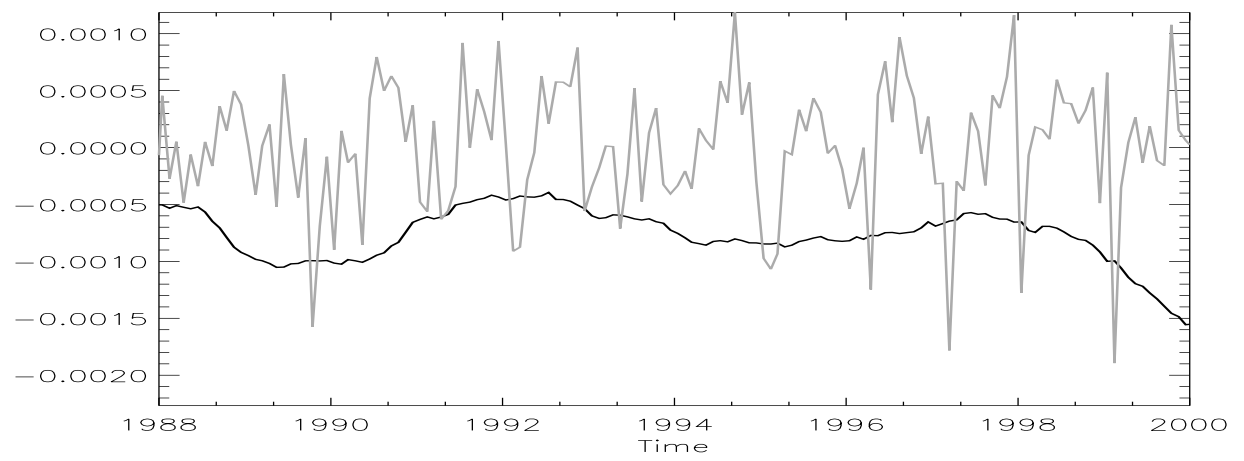

Figure 2. Time evolution of the low and high frequency components of the assimilation increment from $A_{-} S 2$ in the Niño 3 area at $100 \mathrm{~m}$ depth. The 24 -month running mean is shown in black and the high-frequency residuals are shown in grey. Units are ${ }^{\circ} \mathrm{C} /$ hour.

small compared to the high frequency component and that the low frequency component is not constant in time. Particularly noticeable is the negative trend after 1998. The changes in the bias may be due to changes (local or remote) in the observation coverage (introduction of the TRITON moorings in the Western Pacific, for instance). They could also be due to the flow-dependent nature of the error: during the cold phase of ENSO (that started at the end of 1998) the slope of the thermocline is very pronounced, which may be difficult to simulate with a model that tends to produce a flatter-than-observed thermocline (see discussion in section 4). Or they could be caused by changes in the surface fluxes associated with changes in the atmospheric analysis. More work is needed to understand 
these trends, but in any case, figure 2 highlights the non-stationarity of the bias. Ideally, a bias correction algorithm should take this into account.

\section{(c) System Bias and Interannual Variability}

In practice, the presence of system bias may lead to spurious temporal variability in regions where the observation coverage is not uniform in time, which may be a serious problem when the ocean analysis is used to represent interannual variability.

Figure 3a shows the time evolution of the sea level in the equatorial Atlantic $\left(70^{\circ} \mathrm{W}-30^{\circ} \mathrm{E}, 5^{\circ} \mathrm{N}-5^{\circ} \mathrm{S}\right)$ from $A_{-} S 1$ (black line). The most striking feature is the sudden decrease in the sea level around 1985. The $C_{-} S 1$ (not shown) does not exhibit any particular anomaly during that time. An inspection of the time evolution of the observations used in this analysis*, shown in fig $3 \mathrm{~b}$, reveals a sudden increase in the number of observations in the equatorial Atlantic that were assimilated around January 1985. Other (smaller) sea level changes apparent in the $A_{-} S 1$ run occur when the observation coverage changed: both the increase in the number of observations around 1992 and the appearance of PIRATA moorings around 1998 are associated with a decrease in the sea level of the equatorial Atlantic. The latter was reported by Segschneider et al. (2000).

The sudden jump in sea level in the $A_{-} S 1$ run in figure 3 a is a side effect of the data assimilation. The data corrects for a large error in temperature due to a very diffuse thermocline (not shown). The correction requires a large negative increment to the temperature field. Without the corresponding balance correction to the salinity field, the vertical stability of the water column is disrupted, and the assimilation induces spurious convection. In $A \_S 1$ the salinity was not corrected after temperature assimilation, whereas in $A_{-} S 2$ it was.

The grey curve in fig 3a shows the sea level evolution of a prototype of $S 2$, where the salinity is updated by applying conservation of the background state TS relationship. The abrupt jump of the sea level in 1985 is aleviated, but changes in the sea level associated with the evolution of the observing system are still noticeable. If changes in the observing system are very sudden, it may be helpful to have an a priori estimate of the bias in the system, as will be discussed in section 4 .

\section{The Generalized BIAS-CORRECTION ALGORITHM}

(a) Two-step generalized bias-correction algorithm

The standard procedure to deal with systematic error in a data assimilation system is to augment the model state with a set of systematic error or bias variables. Let $\mathbf{x}$ represent a vector belonging to the $n$-dimensional vector space $\mathcal{X}$, and $\boldsymbol{\beta}$ is the vector of bias variables, belonging to the $r$-dimensional bias space $\mathcal{B}$. Let us define $\mathbf{b}(\boldsymbol{\beta})$ as transformation from $\mathcal{B}$ into $\mathcal{X}$ that relates the bias variables with the state vector variables. Following Dee (2005), the generalized expression for the $k^{\text {th }}$ analysis cycle of a data assimilation system is:

* More comprehensive historical observational data sets are now available, such as that prepared as part of the ENACT project (Ingleby and Huddleston 2006), used in section 4. 

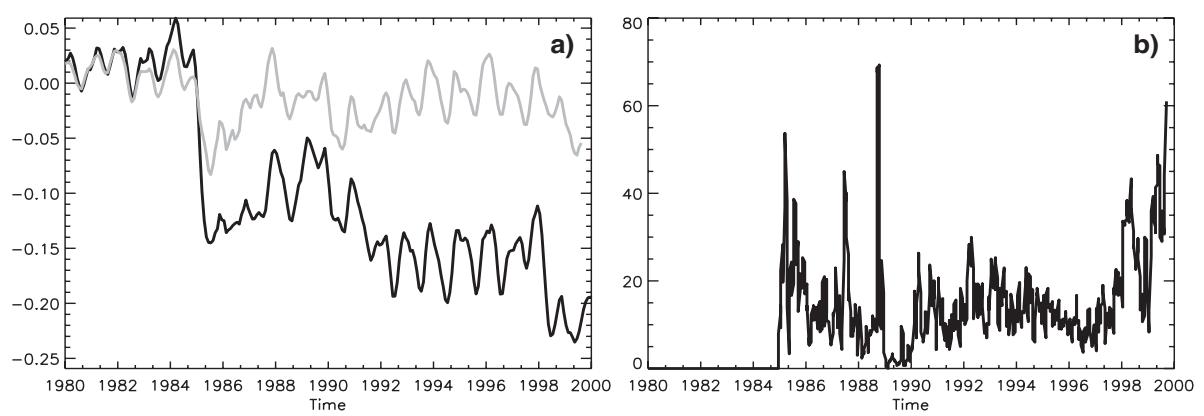

Figure 3. Time evolution of a) the sea level (in metres) averaged over the equatorial Atlantic (5N-5S), as represented by the $A \_S 1$ (black line) ocean analysis $(S 1)$ and by an ocean analysis prototype of $S 2$, where conservation of water masses characteristics is imposed (grey line). b) Time evolution of the number of observations over the same region used in the analysis.

$$
\begin{aligned}
\boldsymbol{\beta}_{k}^{a} & =\boldsymbol{\beta}_{k}^{f}-\mathbf{K}_{\beta}\left[\mathbf{y}_{k}^{o}-\mathbf{H}\left(\mathbf{x}_{k}^{f}-\mathbf{b}_{k}^{f}\right)\right] \\
\mathbf{x}_{k}^{a} & =\left(\mathbf{x}_{k}^{f}-\mathbf{b}_{k}^{a}\right)+\mathbf{K}_{\mathbf{x}}\left[\mathbf{y}_{k}^{o}-\mathbf{H}\left(\mathbf{x}_{k}^{f}-\mathbf{b}_{k}^{a}\right)\right]
\end{aligned}
$$

where the superscripts $f$ and $a$ refer respectively to the forecast and analysis of a given variable, $\mathbf{y}_{k}^{o}$ is the observation vector, and $\mathbf{b}_{k}^{a}$ and $\mathbf{b}_{k}^{f}$ are the abbreviated notations for $\mathbf{b}\left(\boldsymbol{\beta}_{k}^{a}\right)$ and $\mathbf{b}\left(\boldsymbol{\beta}_{k}^{f}\right)$ respectively. To define the terms $\boldsymbol{\beta}_{k}^{f}$ and $\mathbf{b}_{k}^{f}$ we need to prescribe a model for the time evolution of the bias. This will be discussed in section 3(c).

In the special case when $\mathbf{b}$ is linear in $\boldsymbol{\beta}$, i.e.:

$$
\mathbf{b}_{k}^{a}=\mathbf{b}\left(\boldsymbol{\beta}_{k}^{a}\right)=\mathbf{L} \boldsymbol{\beta}_{k}^{a},
$$

and assuming that the observations are unbiased and that forecast and observation errors are uncorrelated, Dee (2005) shows that the optimal gain matrices $\mathbf{K}_{\beta}$ and $\mathbf{K}_{\mathbf{x}}$ for the bias and the state respectively are

$$
\begin{aligned}
\mathbf{K}_{\beta} & =\mathbf{B}_{\beta} \mathbf{L}^{T} \mathbf{H}^{T}\left[\mathbf{H}\left(\mathbf{B}_{\mathbf{x}}+\mathbf{L} \mathbf{B}_{\beta} \mathbf{L}^{T}\right) \mathbf{H}^{T}+\mathbf{R}\right]^{-1} \\
\mathbf{K}_{\mathbf{x}} & =\mathbf{B}_{\mathbf{x}} \mathbf{H}^{T}\left[\mathbf{H B}_{\mathbf{x}} \mathbf{H}^{T}+\mathbf{R}\right]^{-1}
\end{aligned}
$$

where $\mathbf{B}_{\mathbf{x}}$ and $\mathbf{B}_{\beta}$ are the error covariance matrices for the forecasts of the unbiased state and for the bias vector estimates respectively, and $\mathbf{R}$ is the observation error covariance matrix. The original DdS bias correction algorthim is a particular case of the above, where $\mathbf{L}$ is taken as the identity, so that $\mathbf{b} \equiv \boldsymbol{\beta}$.

Equation (1) requires two analysis steps: one for the bias estimation and a second for the state vector. Assuming that the bias is nearly constant in time, and that the bias error covariance matrix is proportional to the forecast error covariance matrix, with the proportionality constant $\gamma$ small compared to one, the algorithm can be approximated so it only requires one analysis step, and thus the bias term can be updated at little extra cost (Radakovitch et al. (2001); Dee (2005)). However the requirement of proportionality between the bias and forecast error covariance matrices is not reasonable in the general case, where the bias 
and the model state vector have different control variables and/or multivariate balance relationships. In the following we derive an alternative approximation for the one-step bias correction algorithm for the general case.

\section{(b) Generalized one-step bias-correction algorithm}

Reduction to a one-step algorithm can be obtained by approximating the bias parameter update

$$
\mathbf{d} \boldsymbol{\beta}_{k}=-\mathbf{K}_{\beta}\left[\mathbf{y}_{k}^{o}-\mathbf{H}\left(\mathbf{x}_{k}^{f}-\mathbf{b}_{k}^{f}\right)\right]
$$

in terms of the unbiased state increment

$$
\mathbf{d x}_{k}=\mathbf{K}_{\mathbf{x}}\left[\mathbf{y}_{k}^{o}-\mathbf{H}\left(\mathbf{x}_{k}^{f}-\mathbf{b}_{k}^{a}\right)\right]
$$

which is already computed in the assimilation algorithm. If the bias is slowly varying in time, i.e.

$$
\left\|\boldsymbol{\beta}_{k}^{a}-\boldsymbol{\beta}_{k}^{f}\right\| \ll\left\|\boldsymbol{\beta}_{k}^{a}\right\|,
$$

then $\mathbf{b}_{k}^{a}$ can be approximated by $\mathbf{b}_{k}^{f}$ so that the terms in the square brackets in eq (4) and eq (5) become identical. In this case, it is also reasonable to assume that the bias estimation errors are much smaller than the background errors, i.e.

$$
\left\|\mathbf{L} \mathbf{B}_{\beta} \mathbf{L}^{T}\right\| \ll\left\|\mathbf{B}_{\mathbf{x}}\right\| \text {. }
$$

Therefore, the matrix terms in square brackets in eq (3) are nearly identical. Note that approximations in the error covariances are always made in practice (Weaver et al. 2005). As a result the bias estimation will be suboptimal but still consistent; see Dee and Todling (2000). Finally, we write

$$
\mathbf{K}_{\beta}=\gamma \mathbf{G K}_{\mathbf{x}}
$$

with $\gamma$ a scalar $\ll 1$, and $\mathbf{G}$ a linear transformation which maps model state into bias parameter space. Comparing with eq (3), eq (8) implyies a covariance model for the bias parameter estimates which satisfies

$$
\mathbf{B}_{\beta} \mathbf{L}^{T}=\gamma \mathbf{G B}_{\mathbf{x}} .
$$

Let us now introduce the following variables for easy notation:

$$
\begin{aligned}
\tilde{\mathbf{x}}_{k}^{f} & =\mathbf{x}_{k}^{f}-\mathbf{b}_{k}^{f} \\
\mathbf{d} \tilde{\mathbf{x}}_{k} & =\mathbf{K}_{\mathbf{x}}\left[\mathbf{y}_{k}^{o}-\mathbf{H} \tilde{\mathbf{x}}_{k}^{f}\right],
\end{aligned}
$$

where $\tilde{\mathbf{x}}_{k}^{f} \approx \mathbf{x}_{k}^{f}-\mathbf{b}_{k}^{a}$ and $\mathbf{d} \tilde{\mathbf{x}}_{k} \approx \mathbf{d x}_{k}$. With this notation, and the approximations in eq (6), eq (7) and eq (9), we can approximate the generalized two step algorithm in eq (1) by a generalized one-step algorithm (G1S) as follows:

$$
\begin{aligned}
\mathbf{x}_{k}^{a} & =\tilde{\mathbf{x}}_{k}^{f}+\mathbf{d} \tilde{\mathbf{x}}_{k} \\
\boldsymbol{\beta}_{k}^{a} & =\boldsymbol{\beta}_{k}^{f}-\gamma \mathbf{G d} \tilde{\mathbf{x}}_{k}
\end{aligned}
$$

In the G1S scheme, the increments to the bias parameters are derived from the state increment $\mathbf{d} \tilde{\mathbf{x}}_{k}$, and therefore only one analysis step is needed. Furthermore, the relationship $\mathbf{G}$ between the bias and the state increment does 
not need to be diagonal, which introduces the possibility of different control variables and/or multivariate relationships for the bias and state vector. In this way, the generalized one step scheme encompasses naturally the BMN scheme (although in the general BMN scheme the operators $\mathbf{G}$ and $\mathbf{L}$ are non linear). For instance, in the system described in section 2, the control variable of the bias vector would be the pressure gradient, while the state vector consists of the $3 \mathrm{D}$ temperature, salinity, velocity and sea level fields $(\mathrm{T}, \mathrm{S}, \vec{U}, \eta)$. The pressure gradient correction $\left(\boldsymbol{\beta}_{k}^{a}\right)$ is derived from the analysis increments in temperature $\left(\mathbf{d} \tilde{\mathbf{x}}_{k}\right)$ via the operator $\mathbf{G}$. The final temperature bias $\mathbf{b}_{k}^{a}$, resulting from the operator $\mathbf{L}$ in eq (2) acting on $\boldsymbol{\beta}_{k}^{a}$, is the effect of applying the pressure gradient correction to the momentum equations and integrating the model forward.

\section{(c) A prediction model for the forecast bias}

We have seen in section 2 that the bias term evolves in time, although slowly. The hypothesis of constant bias could be relaxed to allow for the slow time evolution of the bias without incurring large errors. Here we propose a simple parametric model for the time evolution of the bias. The rational behind this simplification is to avoid having to fit a large number of degrees of freedom with a limited number of observations, while still retaining some flexibility. In $\mathcal{X}$ space, we assume the bias term $\mathbf{b}_{k}^{f}$ evolves in time according to the following model:

$$
\begin{aligned}
\boldsymbol{\beta}_{k}^{f} & =\alpha \boldsymbol{\beta}_{k-1}^{a} \\
\mathbf{b}_{k}^{\prime f} & =\mathbf{L} \boldsymbol{\beta}_{k}^{f} \\
\mathbf{b}_{k}^{f} & =\overline{\mathbf{b}}+\mathbf{b}_{k}^{\prime f} .
\end{aligned}
$$

The forecast bias is represented as the sum of two terms: a prescribed bias term $\overline{\mathbf{b}}$, estimated a priori, and a departure $\mathbf{b}_{k}^{\prime f}$ from $\overline{\mathbf{b}}$. Only the departure $\mathbf{b}_{k}^{\prime f}$ is derived from the on-line estimation $\boldsymbol{\beta}_{k}^{f}$. The on-line bias estimation has finite memory, controlled by the factor $\alpha$. The introduction of the memory term will limit the influence in time of isolated or sporadic observations. It is a way of accounting for uncertainty in the estimation of the bias term, which is proportional to the age of the observations and it also has the potential to allow for time-dependent bias. A side effect is that values of $\alpha$ less than 1.0 will underestimate the magnitude of the bias. To compensate for that, the constant term $\overline{\mathbf{b}}$ is introduced in (12).

The term $\overline{\mathbf{b}}$ is not affected by the on-line estimation and has to be estimated a priori, preferably with independent information. If there is not enough information for independent estimation, it can always be set to zero. Apart from compensating for the damping effects of the memory term, the inclusion of the a priori bias term offers other practical advantages. For instance, it provides a way to extrapolate into the past the information given by more recent observing systems, as opposed to the bias estimated on line $\boldsymbol{\beta}_{k}^{a}$, which only uses past information. This is useful not just for interpreting climate signals in ocean reanalyses, but also for seasonal forecasting, since the reanalyses are used for initial conditions. Thus, the a priori term has the potential to provide a smoother analysis by preventing abrupt changes in the analysis associated with the introduction of new observing systems. The prescribed term could also represent systematic errors in, for example, the seasonal cycle. Finally, the a priori estimation may contain information about other variables or balance relationships not easy to 
estimate through the on-line procedure. For simplicity, in eq (12), there is no explicit reference to the choice of multivariate relationship for the a priori bias term $\overline{\mathbf{b}}$ which belongs to the space vector state $\mathcal{X}$. For the on-line bias term, eq (12) retains the dual representation $\mathbf{b}_{k}^{\prime(f, a)}, \boldsymbol{\beta}_{k}^{(f, a)}$ for the bias in $\mathcal{X}$ and $\mathcal{B}$ vector space respectively.

\section{(d) Implementation of the G1S algorithm}

The implementation of the G1S algorithm makes use of an auxiliary variable $\hat{\mathbf{b}}_{k}^{a}$ belonging to the state vector space $\mathcal{X}$, from which the bias parameters are derived as:

$$
\begin{aligned}
\hat{\mathbf{b}}_{k}^{f} & =\alpha \hat{\mathbf{b}}_{k-1}^{a} \\
\hat{\mathbf{b}}_{k}^{a} & =\hat{\mathbf{b}}_{k}^{f}-\gamma \mathbf{d} \tilde{\mathbf{x}}_{k} \\
\boldsymbol{\beta}_{k}^{a} & =\mathbf{G} \hat{\mathbf{b}}_{k}^{a} .
\end{aligned}
$$

From eq (12) and eq (13) it follows that:

$$
\begin{aligned}
\boldsymbol{\beta}_{k}^{f} & =\mathbf{G} \hat{\mathbf{b}}_{k}^{f} \\
\mathbf{b}_{k}^{f} & =\overline{\mathbf{b}}+\mathbf{L} \mathbf{G} \hat{\mathbf{b}}_{k}^{f} .
\end{aligned}
$$

It is important to notice that in the general case the term $\hat{\mathbf{b}}_{k}^{f}$ (used to estimate the bias parameters in eq (13)) is different from the term $\mathbf{b}_{k}^{f}$ (used to remove the bias from the state vector in eq (12)), since LG does not need to be the identity. This difference can be illustrated taking as an example the BMN scheme as implemented in the ECMWF system. In the BMN scheme, the term $\hat{\mathbf{b}}_{k}^{f}$ is temperature, estimated from the temperature analysis increment. However, $\hat{\mathbf{b}}_{k}^{f}$ does not affect directly the temperature in the model. Rather it is used to estimate $\boldsymbol{\beta}_{k}^{f}$ which is a correction to the pressure gradient. This then affects temperature through the model equations, which in the bias algorithm will be embedded in operator $\mathbf{L}$.

Using equations (10)-(14), the algorithmic implementation of the G1S scheme becomes:

$$
\begin{aligned}
\tilde{\mathbf{x}}_{k}^{f} & =\mathbf{x}_{k}^{f}-\left(\overline{\mathbf{b}}+\alpha \mathbf{L G} \hat{\mathbf{b}}_{k-1}^{a}\right) \\
\mathbf{d} \tilde{\mathbf{x}}_{k} & =\mathbf{K}_{\mathbf{x}}\left[\mathbf{y}_{k}^{o}-\mathbf{H} \tilde{\mathbf{x}}_{k}^{f}\right] \\
\mathbf{x}_{k}^{a} & =\tilde{\mathbf{x}}_{k}^{f}+\mathbf{d} \tilde{\mathbf{x}}_{k} \\
\hat{\mathbf{b}}_{k}^{a} & =\alpha \hat{\mathbf{b}}_{k-1}^{a}-\gamma \mathbf{d} \tilde{\mathbf{x}}_{k} .
\end{aligned}
$$

In (15) it is clear that the estimation of the bias will depend on the values of parameters $\alpha$ and $\gamma$ that determine the time evolution of the on-line bias term, by controlling its memory and amplitude respectively. The bias estimation also depends on the prescription of $\overline{\mathbf{b}}$ and $\mathbf{G}$. In section 4 we conduct a set of experiments to evaluate the sensitivity of the solution to these parameters. When discussing the results we will refer to the variable $\hat{\mathbf{b}}_{k}^{f}$ in eq (13), since in practice the term $\mathbf{L} \mathbf{G} \hat{\mathbf{b}}_{k}^{f}$ is not computed explicitly. For instance, in the BMN scheme, the pressure correction is added to the model pressure gradient in the momentum equations, so that only one integration of the model equations is needed. 
TABLE 1. SUMMARY OF EXPERIMENTS CONDUCTED

\begin{tabular}{|c|c|c|c|c|}
\hline Experiment & time-decay $\sim(1-\alpha)^{-1}$ & $\gamma$ & $\mathbf{G}(T, S, \nabla P)$ & $\mathbf{b}(T, S, \nabla P)$ \\
\hline$E 0$ & & 0 & $(0,0,0)$ & $(0,0,0)$ \\
\hline$P_{M}$ & 2 Years & 0.1 & $(0,0, \nabla P)$ & $(0,0,0)$ \\
\hline$T_{M}$ & 2 Years & 0.1 & $(T, 0,0)$ & $(0,0,0)$ \\
\hline$T S_{M}$ & 2 Years & 0.1 & $(T, S, 0)$ & $(0,0,0)$ \\
\hline$P_{M F}$ & 2 Years & 0.3 & $(0,0, \nabla P)$ & $(0,0,0)$ \\
\hline$P_{I F}$ & infinite $(\alpha=1)$ & 0.3 & $(0,0, \nabla P)$ & $(0,0,0)$ \\
\hline$P_{M \bar{b}}$ & 2 Years & 0.1 & $(0,0, \nabla P)$ & $(T, S, 0)$ \\
\hline
\end{tabular}

Experiment $E 0$ is a standard data assimilation analysis, without bias correction. In all the other experiments there is bias correction, their names according to the following convention: the main one or two letters indicate the choice of $\mathbf{G}$ (pressure gradient $(\nabla P)$, temperature $(\mathrm{T})$, or temperature and salinity (TS)); the subindices indicate the choice of model for the time evolution of the bias: the memory term is indicated by $\mathrm{M} / \mathrm{I}$ in the first subindex, for limited/infinite memory respectively. A subindex $F$ indicates the faster adaptation of bias, corresponding with larger values of $\gamma$. A subindex $\bar{b}$ is indicative of a prescribed component in the bias.

\section{(e) Observability of the bias variables}

The robust on-line estimation of the bias in the G1S scheme depends on properties of the operators $\mathbf{L}$ and $\mathbf{G}$. From eq (11) the bias parameter updates are restricted to the range of $G(\mathbf{d} \boldsymbol{\beta} \in \Re(\mathbf{G}))$, while eq (10) implies that the forecast bias corrections are constrained by the range of $\mathbf{L G}(\mathbf{d} \mathbf{b} \in \Re(\mathbf{L G}))$. Furthermore, the observability of the bias variables will be determined by the rank of HLG. To illustrate this point more explicitly, let us focus on the particular case $\overline{\mathbf{b}}=\mathbf{0}$. In this case, the assimilation increment for the state vector, in terms of $\hat{\mathbf{b}}$, takes the form:

$$
\mathbf{d} \tilde{\mathbf{x}}_{k}=\mathbf{K}_{\mathbf{x}}\left[\mathbf{y}_{k}^{o}-\mathbf{H} \mathbf{x}_{k}^{f}+\mathbf{H L G} \hat{\mathbf{b}}_{k}^{f}\right] .
$$

This relation shows the coupling between the bias and the state vector variables: If the image of operator $\mathbf{L}$ lies in the null space of $\mathbf{H}$, or if $\hat{\mathbf{b}}_{k}^{f}$ lies in the null subspace of $\mathbf{L G}$, there will no feedback between the bias variables and the observations. The null space of $\mathbf{L G}$ are those directions of the state vector that either are not affected by the bias (i.e. do not belong to the image of $\mathbf{L}$ ) or that do not influence the estimation of $\boldsymbol{\beta}_{k}^{a}$ (i.e., belong to the null space of $\mathbf{G}$ ).

\section{Sensitivity experiments}

\section{(a) Experimental setup}

For the sensitivity experiments we use an up-to-date version of the data assimilation system described in section 2 . The observations come from the more comprehensive and quality-controlled data set produced as part of the ENACT project (Ingleby and Huddleston 2006). The forcing fields are derived from ERA40, with the modifications in the fresh water introduced by Troccoli and Kallberg (2004). The version of the ocean model is the same as for S2, but at lower resolution ( $2 \times 2$ degrees lat/lon with equatorial refinement). All the experiments start from the same spin up and span the period January 1987 - December 2001. Only subsurface temperature data are assimilated, but salinity and currents are updated through the multivariate relationships described in section 2 .

Table 1 shows the summary of the different experiments. Experiment E0 was conducted as a control, with standard assimilation and no bias correction. Three experiments were conducted to test the sensitivity of the results to the multivariate formulation of the bias: in experiment $P_{M}, \mathbf{G}$ was chosen to simulate 
the BMN scheme, i.e. the information from the temperature observations is used to correct the bias in the pressure gradient, but no explicit correction is made to the bias in temperature; in experiment $T_{M}$, only the bias in the temperature field is corrected explicitly, as in Chepurin et al. (2005), with no modifications to the bias pressure fields; and in experiment $T S_{M}$, the biases in both the temperature and salinity fields are corrected (although only observations of $T$ are used). In all these experiments the bias term has finite memory (with the value of $\alpha$ equivalent to 2 -years time-decay). There is no definitive way to set the value of $\gamma$, and in the above experiments we chose a value of $\gamma=0.1$.

An additional set of experiments was conducted to test the sensitivity to the parameters $\alpha$ and $\gamma$ controlling the time evolution and amplitude of the bias. In these experiments the bias is applied to the pressure gradient, as in $P_{M}$. In experiment $P_{M F}$ the value of $\gamma$ is increased to 0.3 (i.e. the bias adapts faster) but the value of $\alpha$ is still equivalent to a time-decay of 2 years. In experiment $P_{I F}, \alpha$ is set to 1.0 , so the observations will influence the bias estimate indefinitely, and $\gamma=0.3$. In all the above experiments $\left(P_{M}, T_{M}, T S_{M}, P_{M F}\right.$ and $\left.P_{I F}\right) \overline{\mathbf{b}}$ is zero.

Finally, experiment $P_{M \bar{b}}$ was used to evaluate the importance of the prescribed bias. The term $\overline{\mathbf{b}}$ contained modifications to the temperature and salinity fields, and zero correction to the pressure gradient. The term was derived from a climatological model run, where the ocean model was forced by climatological ERA-40 fluxes and relaxed to the WOA98 climatology (Levitus et al. 1998) with a time scale of 3 years. The $\overline{\mathbf{b}}$-term was estimated as the annual mean of the corrections due to the WOA98.

\section{(b) Sensitivity to the multivariate formulation}

The left column of figure 4 shows the 1987-2001 average of a longitude-depth section of the assimilation increments along the equator from experiments E0, $T_{M}$ and $P_{M}$ (panels a, b and c respectively). The mean increment in fig 4a has a large-scale dipolar structure in the equatorial Pacific, as if the data assimilation were correcting the slope of the thermocline, making it deeper in the western Pacific and shallower in the eastern Pacific. This kind of error could appear if the equatorial winds were too weak, although it may be due to other mechanisms. In section 2, it was suggested that the negative increment in the Eastern Pacific is in fact partly induced by the assimilation process. In the experiments $T_{M}$ and $P_{M}$, where the bias has been corrected online, the resulting mean increment is smaller (though it is not removed entirely since $\alpha<1$.), both in the eastern and western Pacific. In $T_{M}$, the reduction of the mean increment in temperature is expected since the bias correction acts directly in T. However, as will be shown below, the smaller mean increment in temperature does not guarantee a better analysis.

For a more impartial test of the performance of the bias correction algorithms we need to look at independent variables. The right column of figures 4 shows an equatorial cross-section of the vertical velocity for the respective experiments in the left column. The spurious vertical circulation in the Eastern Pacific associated with the degradation of the zonal current discussed in section 2 is evident in panel d. Correcting the bias only in temperature degrades it even further (fig 4e). The behaviour is similar to, and consistent with, that observed in experiments where the weight given to the observations is increased: the equatorial currents are systematically degraded (not shown). If a bias correction algorithm is used, the 

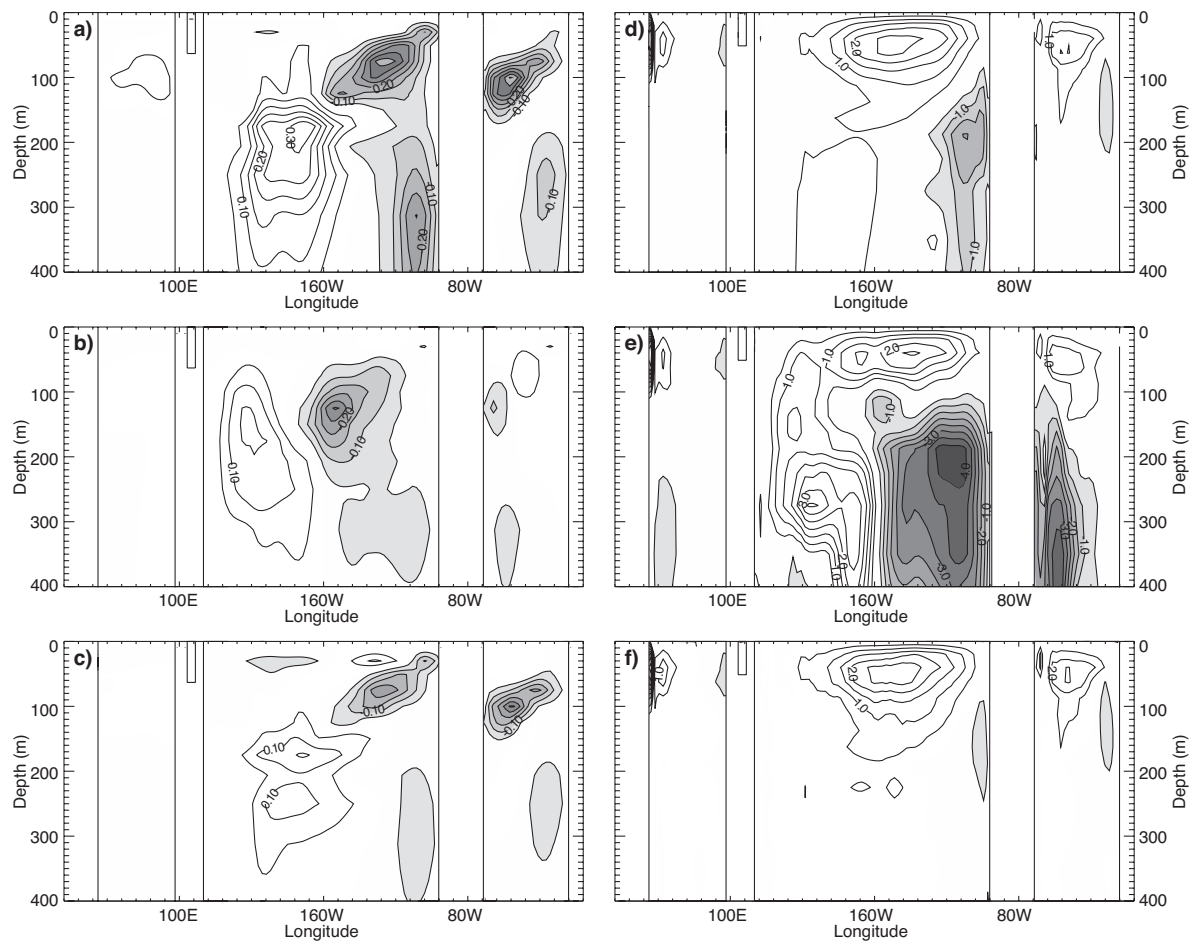

Figure 4. Equatorial longitude-depth section of mean temperature increment (left column) and vertical velocity (right column) for experiments $E 0$ (a and d), $T_{M}$ (b and e) and $P_{M}$ (c and f). Contours every $0.5^{\circ} \mathrm{C} / 10$-days for the temperature increment and $0.5 \mathrm{~m} /$ day for the vertical velocity. The zero contour is not plotted, and shading represents negative values. The mean corresponds to the time average during the period 1987-2001.

observations are indirectly given more weight, since they are allowed to influence the estimate twice: directly through the difference $\mathbf{y}_{k}^{o}-\mathbf{H x}_{k}^{f}$, and indirectly through the bias. The latter allows the data to have a longer lasting influence.

In the experiment where the bias is treated by applying a correction to the pressure gradient using the BMN scheme (fig 4f), the spurious circulation does not appear. This result highlights the merits of the BMN scheme, and illustrates large sensitivity of the results to the choice of multivariate relationship in the operator $\mathbf{G}$ in (11). As pointed out by Burgers et al. (2002), the bias may have its origins in the momentum equation (resulting from inaccuracies in the wind field and in the vertical mixing of momentum among others). If so, the error should be "adiabatic", since it is due to the wrong redistribution of heat. The BMN is a way of imposing adiabaticity in the assimilation of temperature data, by assuming that the bias arises entirely from an incorrect value in the pressure gradient terms, and using the temperature increments given by the assimilation to derive a correction to the pressure gradient.

It can be argued that in experiment $T_{M}$, where the bias acts only on temperature, no balance corrections are made to the bias in salinity, and there is the potential of disrupting the water mass characteristics. (Ricci et al. (2005) show that the impact of salinity on the equatorial velocity field is not negligible). In experiment $T S_{M}$, the bias in salinity is estimated by setting $\mathbf{G}=\mathbf{L}=\mathbf{I}$ in eq 
(15)). Hence, the salinity bias is the running mean of the salinity increments $\mathbf{d} \tilde{\mathbf{x}}_{k}$ for the state vector, derived from the local T-S relationship and the unbiased temperature increment. Results (not shown) were very poor, with marked trends in salinity and sea level. In this case the bias algorithm failed to stabilize the salinity field, probably because the bias in salinity can not be constrained by the temperature observations. A possible reason for the poor results in experiment $T S_{M}$ may lie in the nonlinear nature of the T-S relationship: the bias in $\mathrm{S}$ obtained by accumulating the salinity increments of the independent analysis cycles is not the same as if the nonlinear T-S relationship were computed using the bias in T. The G1S algorithm could still be used to impose preservation of water mass characteristics for the bias term, but has not been tested and further work is needed for its correct implementation.

\section{(c) Sensitivity to the parameters in the time evolution model}

Figure 5 shows results from experiments conducted to evaluate the sensitivity to the time evolution of the bias term. Although in the experiments discussed here the bias correction is only applied in the pressure gradient, the bias in temperature used to derive the pressure correction $\left(\hat{\mathbf{b}}_{k}^{f}\right.$ in eq $\left.(13)\right)$ is shown. In the left column, the time evolution of the estimated $\hat{\mathbf{b}}_{k}^{f}$ is shown at three different depths over the region Niño 3. The time evolution of the 24-month running mean of the temperature increment is shown in the right column, together with the value of the mean assimilation increment (shaded ticks on the right y-axis). Because of the 24-month running mean, the time axis in these graphs is limited to the period 1989-2000. The black solid line is for experiment $P_{M}$, the grey solid line is for experiment $P_{I F}$ and the grey dashed line is for experiment $P_{M F}$. For reference, the thin black line shows the value that the bias would have had in experiment $E 0$, had it been estimated using the same parameters as in $P_{M}$ (remember that the online bias correction is not active in $E 0$, i.e. $\mathbf{G}=0$ ). The resulting values and behaviour of the bias estimates are very different in the different experiments. In the following section we try to use these diagnostics to assess the quality of the resulting analyses.

One possible criterion for assessment is to require that the estimated bias be consistent with the model prescribed for the time evolution of the bias. For instance, if the assumption is constant bias, then the resulting estimate should exhibit convergence to a constant value: after this value is reached, the analysis increment should be white noise with zero mean. In general, the time average of the temperature increment should be as close as possible to zero.

At $30 \mathrm{~m}$ depth (fig 5a), the bias in experiment $P_{I F}$ (which assumes constant bias) does not converge. The bias keeps increasing with time, and the resulting value is of the opposite sign to the reference experiment $E 0$ but with much larger amplitude. The 24-month running mean assimilation increments (fig 5b) are mainly positive, and after 1996 exhibit large fluctuations in time. The value of the mean temperature increment in $P_{I F}$ is positive and has the largest absolute value. The resulting velocity field is too weak compared to observations (not shown). It can be concluded that at least for this region and level, the time evolution of bias diagnosed a posteriori for experiment $P_{I F}$ is not consistent with the model for the bias correction term prescribed a priori ( $\alpha=1$ in eq (12) would imply constant bias); it seems as if the bias is overcorrected, resulting in a change of sign. It is not clear at this point if the pathological behaviour of $P_{I F}$ is caused 

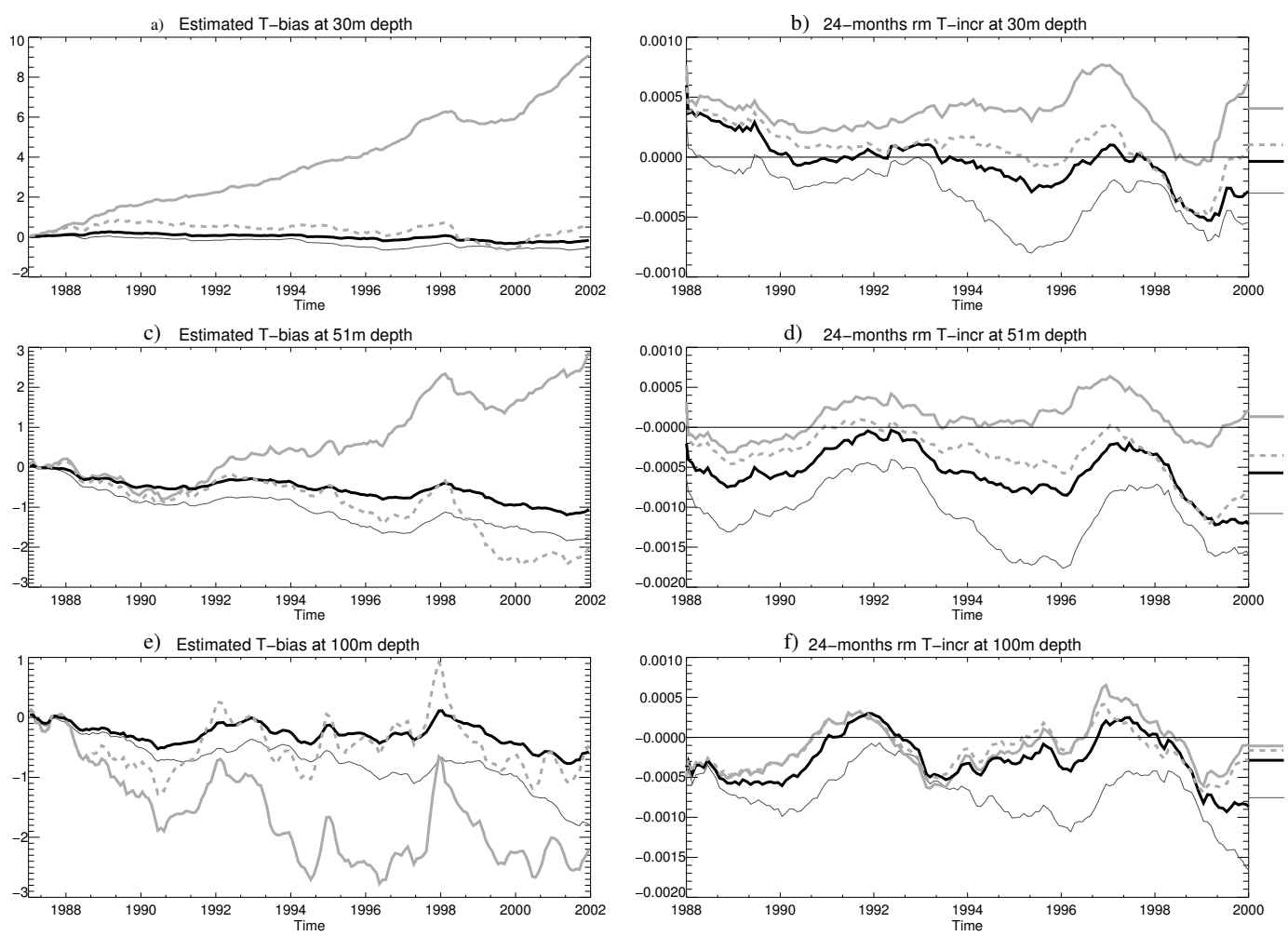

Figure 5. Time evolution of the estimated bias, $\hat{\mathbf{b}}_{k}^{f}$ in eq(13), (left column) and 24-month running mean assimilation increment (right column) at different levels in the Niño3 area. Values for the 19882001 average assimilation increment are shown on the y-axis of the right panels. Shown are the results from experiment $P_{M}$ (thick solid black line), $P_{I F}$ (solid grey line), $P_{M F}$ (grey dashed line) and $E 0$ (thin solid back line). In the case of experiment $E 0$ the bias is only a diagnostic; it is not corrected interactively. The bias values are in ${ }^{\circ} \mathrm{C}$ and the assimilation increment in ${ }^{\circ} \mathrm{C} /$ timestep (i.e. ${ }^{\circ} \mathrm{C} / 7200 s$ ).

by the infinite memory $(\alpha=1)$ or by the larger amplitude of the bias (which is a function of $\alpha$ and $\gamma$ ). However, fig 5 a shows that the bias in the experiments with finite memory is more stable; the evolution of the 24-month running mean increment shows that at $30 \mathrm{~m}$ depth both $P_{M F}$ and $P_{M}$ have less bias than $E 0$.

At $50 \mathrm{~m}$ depth, the behaviour of the experiments is more varied. From the point of view of the bias, experiment $P_{I F}$ is still the outlier, and again it overestimates the value of the bias, although not as clearly as at $30 \mathrm{~m}$ depth. In fact, it shows the smallest absolute value of the mean increment. The sensitivity of the results to the parameter $\gamma$ can now be appreciated by comparing the experiments with finite memory: $P_{M}$ where $\gamma$ is 0.1 (black line) to experiment $P_{M F}$, where $\gamma$ is 0.3 (dashed grey line). Consistent with a larger value of $\gamma$, experiment $P_{M F}$ exhibits more time variability, and clearly adapts faster to a large negative value after the change observed in 1998 (and discussed in section 2 ), where it remains until the end of the run. From this graph it is not easy to say if the resulting value of the bias after 1998 is correct. There is a hint that the 24month running mean is going back to zero after 1999, which is consistent with the stabilisation of the bias. In this experiment the high frequency of the assimilation 
increments had smaller variance (not shown). Experiment $P_{M}$ exhibits much smoother temporal behaviour, as expected from the smaller value of $\gamma$. The magnitude of the bias is smaller than that in experiment E0, which reflects the positive impact of the on-line correction. The mean assimilation increment is also smaller than in $E 0$, but larger than in $P_{M F}$. In the two experiments with finite memory the velocity field is improved with respect to the reference experiment E0 (not shown).

At $100 \mathrm{~m}$ depth, in terms of mean absolute error, the best estimator is the experiment with infinite memory. The experiment with fast update $\left(P_{M F}\right)$ follows closely, and experiment $P_{M}$ underestimates the magnitude of the bias (fig $5 \mathrm{f}$ ). Although in some areas $P_{I F}$ is good, it tends to overestimate the bias, sometimes producing quite pathological behaviour, and the experiments with finite memory, as expected, tend to underestimate the bias.

There is no experiment that behaves best in all locations, which suggests that the time parameters may need to be spatially dependent. Without any further theoretical insight, the spatial distribution of parameters $\alpha$ and $\gamma$ could be estimated empirically, together with $\overline{\mathbf{b}}$ (which appears in (15) but has not been discussed yet).

\section{(d) Sensitivity to the prescribed bias}

In order to make good use of recently-developed and future observing systems, such as the ARGO floats, it may be desirable to have an a priori estimate of the bias term. Otherwise, the arrival of new information may induce discontinuities and spurious variability in the analysis of traditionally poorly observed areas. In our simple model for the evolution of the bias (Eq. 12), the $a$ priori bias estimate is given by $\overline{\mathbf{b}}$.

If observations are scarce, $\overline{\mathbf{b}}$ may not be easy to estimate. By gathering all the existing observations in a climatology, such as the WOA98, it would be possible to "gain" spatial coverage by sacrificing the time dimension (and under the strong assumption that the system is stationary). This is roughly the strategy followed here to estimate the term $\overline{\mathbf{b}}$. The ocean model forced by ERA-40 climatology is nudged, with a time scale of 3 years, to the WOA98 climatology. The time scale for relaxation is an ad-hoc way of introducing uncertainty for the WOA98 estimate. There may be more optimal ways of estimating $\overline{\mathbf{b}}$, but we chose a simple one for demonstration purposes. The relaxation terms in the $\mathrm{T}$ and $\mathrm{S}$ equations are taken to provide the estimate of $\overline{\mathbf{b}}$, which would be used to correct T and $\mathrm{S}$ directly. There is no pressure correction in $\overline{\mathbf{b}}$ (see experiment $P_{M \bar{b}}$ in Table 1).

Figure 6 shows the vertical profiles of the 1987-2001 mean assimilation increments for region EQ3 in the Central-Western Pacific $\left(150^{\circ} \mathrm{E}-170^{\circ} \mathrm{W}, 5^{\circ} \mathrm{N}\right.$ $\left.5^{\circ} \mathrm{S}\right)$ and for region EQATL in the Equatorial Atlantic $\left(70^{\circ} \mathrm{W}-30^{\circ} \mathrm{W}, 5^{\circ} \mathrm{N}-5^{\circ} \mathrm{S}\right)$. Shown are the results for experiments $E 0$ (solid back line), $P_{M}$ (black dashed line) and $P_{M \bar{b}}$ (in solid grey). In region EQ3 (left panel of fig 6 ), the term $\overline{\mathbf{b}}$ has visibly reduced the mean temperature increment in the upper ocean. This is a relatively well observed area, where there should be enough observations for the estimation of the on-line pressure correction. In spite of that, the impact of the term $\overline{\mathbf{b}}$ is comparable to the impact of the on-line pressure correction, suggesting that in this area, where the mixed layer is quite deep, the adiabatic corrections are not enough to correct the bias temperature and salinity. 

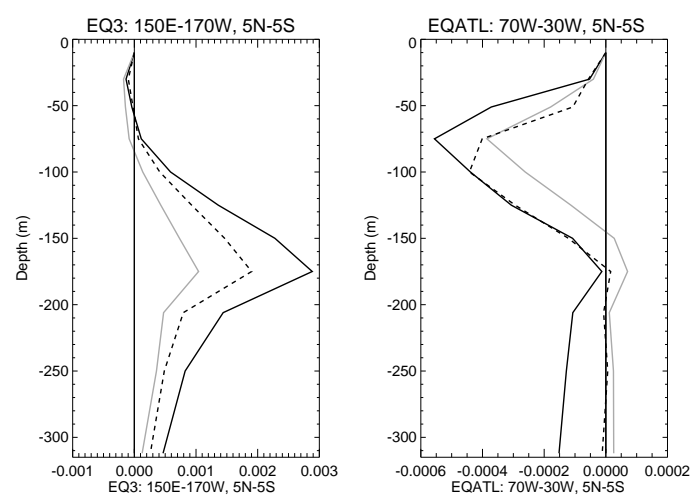

Figure 6. Vertical profiles of the 1987-2001 mean temperature assimilation increment for region EQ3 (left column) and for region EQATL (right column). Shown are the results for experiments $E 0$ (solid back line), $P_{M}$ (black dashed line) and $P_{M \bar{b}}$ (in solid grey). The units in the horizontal axis are ${ }^{\circ} \mathrm{C} /$ timestep

The term $\overline{\mathbf{b}}$ also improves the estimate of the Equatorial Atlantic, both the mean value (reduced mean assimilation increment in right panel of figure 6), and the interannual variability. The quality of the interannual variability can be measured by the correlation of the analysed sea level anomalies with those from the altimeter data. The correlation period is 1993-2001. If no bias correction is applied, the data assimilation degrades the correlation (from 0.65 in an analysis with no data assimilation to less that 0.4 in the experiment $E 0$ ). The inclusion of the on-line correction in pressure in experiment $P_{M}$ slightly improves the estimate, but the correlation is still lower than the no data assimilation case. By introducing the term $\overline{\mathbf{b}}$ in experiment $P_{M \bar{b}}$ the value of the correlation increases to 0.8 . The higher value of the correlation due to the term $\overline{\mathbf{b}}$ is an encouraging result.

As expected, poorly observed regions such as the Equatorial Indian Ocean are better represented if the term $\overline{\mathbf{b}}$ is introduced (in the sense that the mean assimilation increments are reduced). The Equatorial Indian Ocean is only sensitive to the $\overline{\mathbf{b}}$ term, while the on-line correction to pressure has almost no effect (not shown).

\section{Summary and CONClusions}

The presence of bias in an ocean data assimilation scheme is a serious obstacle to the reliable representation of climate by historical ocean reanalysis. This fact, common to other reanalysis systems, is illustrated with examples from the ECMWF operational ocean analysis systems (System 1 and System 2).

In the equatorial Pacific, the mean temperature assimilation increment is different from zero, and shows a large scale dipolar structure. The magnitude of the low frequency component of the temperature assimilation increment (or bias) is comparable to the magnitude of the higher frequency component, indicating that errors in the first guess are correlated in time. The bias is not constant in time, but exhibits some interannual variability.

Consistent with other assimilation systems, comparison with TAO currents shows that the equatorial zonal velocity in the Eastern Pacific is degraded when 
assimilating temperature data, even when salinity is also corrected by imposing preservation of the T-S relationship. The degradation of the zonal velocity is associated with a spurious vertical circulation underneath the thermocline. As pointed out by BMN, the results suggest that the spurious circulation can be related to the bias in temperature: the error in the data assimilation has the opposite sign to the error in an analysis where no data have been assimilated.

Data assimilation systems affected by bias are very vulnerable to changes in the observing system. This fact is illustrated by the large changes in the interannual variability in the Equatorial Atlantic due to the sudden changes in the observation coverage. The sustainability of observing systems is therefore vital for ongoing and future estimates of climate variability. To make optimal use of new and existing observations it is necessary to develop data assimilation algorithms that explicitly deal with bias.

In this paper we have presented a generalized version of the one-step DdS algorithm for on-line estimation and correction of system bias. The modifications, based on Dee (2005), include an explicit multivariate formulation which allows the balance constraints for the bias to be different to those for the state vector. In this context, the correction applied to the pressure gradient proposed in the BMN scheme can be considered as a particular choice of balance relationship. Modifications have also been introduced in the equation for the time evolution of the bias, by inclusion of a memory term that accounts for non-stationary bias, and a prescribed bias term that can act as a first guess. The modified bias correction algorithm has been implemented into the new ECMWF operational ocean data assimilation system (System 3). Various sensitivity experiments have been conducted with different multivariate constraints and values of the parameters controlling time evolution. All the experiments span the period January 1987 - December 2001 and use the ENACT experimental setup. The focus of the discussion is on the equatorial oceans. In all the experiments only temperature data are assimilated.

Results reflect the sensitivity of the analysis to the choice of multivariate formulation. In the Equatorial Pacific, direct univariate correction of the bias in temperature (as in Chepurin et al. (2005)) does not lead to better analyses, and in fact can degrade the equatorial currents by inducing large spurious vertical circulation. However, if the bias is corrected using the BMN scheme by modifying the pressure gradient, the bias in temperature is reduced and the velocity field is improved. Therefore, some insight into the nature of the error is needed for the formulation of the bias error statistics. If the error in temperature is due to adiabatic processes that erroneously redistribute heat, the bias term should reflect this adiabaticity. Otherwise, it will introduce sources and sinks of heat that may degrade the solution. Budget analysis of the assimilation statistics are valuable tool to obtain information about the nature of the error.

The results illustrate that the sensitivities to the parameters controlling the time evolution of the bias are quite large. Generally, experiments with finite memory tend to do better, at the expense of underestimating the size of the bias. The assumption of constant bias, i.e. $\alpha=1$., can lead to overestimation of the bias term and sometimes to pathological results, with the bias term monotonically increasing in time. This is the case in the upper levels of the Eastern Pacific, where the system fails to stabilize, producing estimates that are worse than the nonbias-corrected results. From a practical point of view, underestimation of the bias is probably a safer option, unless the stability of the system is well understood. 
Further work is needed to develop a satisfactory framework for treatment of time dependent bias, or more generally, for treatment of errors at different time scales. There is also a need for well defined metrics that allow us to assess the mathematical consistency and physical validity of the bias correction algorithms.

In order to avoid discontinuities in the ocean analysis due to changing observing systems it would be desirable to have an a priori knowledge of the system bias, preferably obtained using independent data. For instance, the information about the mean dynamic topograpy provided by the gravity missions GRACE and GOCE could in principle provide a first estimate of the bias. Using a prescribed bias term is a way of extrapolating observation information into the past. In this way the geoid information can be exploited in ocean reanalysis even for time periods preceding the gravity missions.

\section{ACKNOWLEDGEMENTS}

The authors want to thank Anthony Weaver and Michael Fisher for their valuable comments, as well as the two reviewers, whose suggestions have greatly improved the manuscript.

Alves. J. O., Balmaseda, M. A., Anderson, D. L. T. and Stockdale, T. N.

Balmaseda, M. A.

Bell, M. J., Martin, M. J., and Nichols, N. K.

Behringer, D. W., Ming Ji, M. and Leetmaa, A.

Burgers, G., Balmaseda, M. A., Vossepoel, F.,van

Oldenborgh, G. J. and van Leeuwen, $\mathrm{P}$.

Carton, J. A.,

Chepurin, G.,Cao, X., and Giese, B. S.

Chen, D., Cane, M. A., Kaplan, A., Zebiak, S. E., and Huang, D.

Chepurin, G. A., Carton, J., and Dee, D. P.

Dee, D. P., and Da Silva, A. M.

Dee, D. P., and Todling, R.

Dee, D. P.

Eden, C., Greatbatch, R. J. and Bning, C. W.

Friedland, B.

\section{REFERENCES}

2004

2004

2004

1998

2002

2000

2004

2005

1998

2000

2005

2004

1969
Sensitivity of dynamical seasonal forecasts to ocean initial conditions. Q. J. R. Meteorol. Soc., 130, 647-668.

Ocean data assimilation for seasonal forecasts. ECMWF Seminar Proceedings. Seminar on Recent developments in data assimilation for atmosphere and ocean, 8-12 September 2003,301-326.

Assimilation of data into an ocean model with systematic errors near the equator. Q. J. R. Meteorol. Soc., 130, 873-893.

An improved coupled model for ENSO prediction and implications for ocean initialisation. Part 1 The Ocean data assimilation system. Mon. Weath. Rev., 126, 10131021.

Balanced ocean-data assimilation near the equator. $J$ Phys Oceanogr, 32, 2509-2519.

A Simple Ocean Data Assimilation analysis of the global upper ocean 1950-1995, Part 2: results J. Phys. Oceanogr., 30, 294-309.

Predictability of El Nino in the past 148 years. Nature,428, 733-736

Forecast model bias correction in ocean data assimilation. Mon. Wea. Rev., 133, 1328-1342

Data assimilation in the presence of forecast bias. Q. J.R. Meteorol. Soc.,124, 269-295.

Data assimilation in the presence of forecast bias: The GEOS moisture analysis. , Mon. Wea. Rev., 128, 3268-3282.

Bias and data assimilation. Q. J. R. Meteorol. Soc. ,131, 3323-3343.

Adiabatically correcting an eddy-permitting model using large-scale hydrographic data: Application to the Gulf Stream and the North Atlantic Current, J. Phys. Oceanogr. , 34, 701-719.

Treatment of bias in recursive filtering. IEEE Trans. Autom. Contr., AC-14, 359-367. 
Gibson, J. K., Kallberg, P., Uppala, S., Nomura, A. and Serrano, E.

Huddleston, M. R., Bell, M. J., Martin, M. J. and Nichols, N. K.

Ingleby, B. and Huddleston, M.

Ji. M., Leetmaa, A. and Derber, J.

Levitus, S., Boyer, T. Conkright, M., Johnson, D., O'Brien, T., Antonov, J., Stephens, C. and Gelfeld, R.

Radakovich, J. D., Houser, P. R., da Silva, A. M., and Bosilovitch, M. G.

Reynolds, R. W., Rayner, N. A., Smith, T. M., Stokes, D. C. and Wang, W.

Ricci. S., Weaver, A. T., Vialard, J. and Rogel, P.

Segschneider, J., Balmaseda, M. A. and Anderson, D. L. T.

Sheng, J., Greatbatch, R. J. and Wright, D. G.

Stammer, D., Wunsh, C., Giering, R., Eckert, C., Heimbach, P., Marotzke, J., Adcroft, A., Hill, C. N. and Marshall, J.

Stammer, D., Ueyoshi, K., Köhl, A., Large, W. G. Josey, S. A. and, Wunsh, C.

Troccoli, A., Balmaseda, M. A., Segschneider, J., Vialard, J., Anderson, D. L. T.,

Haines, K., Stockdale, T. N., Vitart, F. and Fox, A.

Troccoli, A. and Kallberg, P.

Uppala, S., and coauthors

Vialard, J., Weaver, A. T., Anderson, D. L. T. Delecluse, $\mathrm{P}$.

Vialard, J., Vitart, F., Balmaseda, M. A., Stockdale, T. N., and Anderson, D. L. T.

Vidard, A., Piacentini, A., Le Dimet, F.-X

Vidard, A., Anderson, D. L. T. and Balmaseda, M. A.

Weaver, A. T., Vialard, J. and Anderson, D. L. T.

Weaver, A. T., Deltel, C., Machu, E. and Ricci, S.
1997 ERA Description. ECMWF ERA-15 Project Report Series, 1, 71pp.
2004

2006

1995

1998

2001

2002

2005
Assessment of wind stress errors using bias corrected ocean data assimilation. Q. J. R. Meteorol. Soc., 130, 853871.

Quality control of ocean profiles - historical and real-time data.Journal of Marine Systems. In press.

An ocean analysis system for seasonal to interannual climate studies. Mon. Wea. Rev., 123, 460-481.

Introduction. Vol. 1, World Ocean Database 1998, NOAA Atlas NESDIS 18, 346pp.

Results from global land-surface data assimilation methods. 5th Symposium on Integrated Observing Systems, Albuquerque, New Mexico.

An improved in situ and satellite SST analysis for climate. J. Clim, 15, 1609-1625.

Incorporating state-dependent Temperature-Salinity constraints in the background error covariance of variational data assimilation. Mon. Wea. Rev., 133, 317-338

Anomalous temperature and salinity variations in the tropical Atlantic: possible causes and implications for the use of altimeter data. Geophys. Res. Lett., Vol 17, No. 15, p. 2281.

Improving the utility of ocean circulation models through adjustment of the momentum balance. J. Geophys. Res., 106, 16,711-16,728.

The global ocean circulation during 1992-1997 estimated from ocean observations and a general circulation model. J. Geophys. Res., 107 (C9), 3118,doi: 10.1029/2001JC000888.

Estimating air-sea fuxes of heat, fresh water and momentum through global ocean data assimilation. J.Geophys.Res., 109, C05023.

Salinity adjustments in the presence of temperature data assimilation. Mon. Wea. Rev., 130, 89-102.

Precipitation correction in the ERA-40 reanalysis, ERA-40 Project Report Series, 13

The ERA-40 Reanalysis. Q. J. R. Meteorol. Soc.131,Part B, 2961-3012.

Three- and Four-Dimensional Variational Assimilation with a General Circulation Model of the Tropical Pacific Ocean. Part II: Physical Validation. Mon. Wea. Rev., 131, 1379-1995.

An ensemble generation method for seasonal forecasting with an ocean-atmosphere coupled model. Mon. Wea. Rev., 131, 1379-1395. See also ECMWF Technical Memorandum No 417.

Variational Data Analysis with control of the forecast bias, Tellus A, 56 (3), 177-188.

Impact of ocean observation systems on ocean analysis and seasonal forecasts. ECMWF Technical Memorandum No 460.

Three- and Four-Dimensional Variational Assimilation with a General Circulation Model of the Tropical Pacific Ocean. Part I: Formulation, Internal Diagnostics, and Consistency Checks. Mon. Wea. Rev.,131, 1360-1378.

A multivariate balance operator for variational ocean data assimilation. Q. J. R. Meteorol. Soc.,131,3605-3625. 